\title{
Monetizing Online Content: Digital Paywall Design and Configuration
}

\author{
Robert Rußell • Benedikt Berger • Lucas Stich • Thomas Hess • Martin Spann
}

Received: 6 April 2019/Accepted: 6 January 2020/Published online: 12 February 2020

(C) The Author(s) 2020

Keywords Digital paywalls · Online content ·

Monetization $\cdot$ Revenue models $\cdot$ Pricing

\section{Introduction}

The media industry was among the first affected by digitization, because digital technologies have changed content production, distribution, and consumption profoundly. Most media companies have suffered revenue losses, being unable to adapt their monetization strategies to the rapidly changing media consumption patterns of their recipients. Additionally, advertising revenues have proven to be a less reliable revenue source in online than in offline media markets. Consequently, a major obstacle to the content providers' successful digital transformation is the establishment of sustainable revenue streams (Chyi 2012). The

Accepted after two revisions by Ulrich Frank.

R. Rußell $(\varangle) \cdot$ B. Berger · T. Hess

Institute for Information Systems and New Media, Ludwig-

Maximilians-Universität München, Ludwigstr. 28,

80539 Munich, Germany

e-mail: russell@bwl.lmu.de

B. Berger

e-mail: benedikt.berger@bwl.lmu.de

T. Hess

e-mail: thess@bwl.lmu

\section{Stich $\cdot$ M. Spann}

Institute of Electronic Commerce and Digital Markets, Ludwig-

Maximilians-Universität München, Ludwigstr. 28,

80539 Munich, Germany

e-mail: stich@bwl.lmu.de

M. Spann

e-mail: spann@bwl.lmu.de market for music and video content reveals a trend toward direct monetization, with subscription-based streaming services such as Spotify or Netflix showing increasing revenues. However, other media markets, including the market for news content, still struggle to monetize their content online (PricewaterhouseCoopers 2019). After extensive experimentation with various monetization approaches and several failed attempts, more and more news content providers adopt digital paywalls to counter the impending market trends. While the term digital paywall is commonly used in the context of news content, the functionality of this instrument to govern consumers' access to online content is also important for other media products such as music, videos, or games. However, a standard digital paywall does not exist. Owing to the wide range of and new developments in configuration options, the limited experience with digital paywalls in practice, and a lack of research on this topic, it is still unclear which factors determine the optimal design of a digital paywall.

The process of establishing a viable business model for online content is complex and remains a challenge for media companies. Content providers seek to maximize direct revenue by selling content and indirect revenue through advertising (i.e., a two-sided market), while simultaneously considering cannibalization and spillover effects on their traditional formats. Whereas news content providers usually rely on both revenue sources for their printed editions, most of them initially offered their online editions free of charge and only monetized them indirectly through advertising. Although online advertising revenue has been growing steadily, this growth has not been sufficient to compensate for the revenue losses from offline sources such as print subscriptions or advertising. The small margins in online advertising are mainly due to the intensified competition in the digital channel, driven by 
news aggregators, social media networks, and efficiently designed ad exchange platforms. Additionally, the classifieds market, which news content providers traditionally owned, has almost entirely moved toward online platforms such as Craigslist, Indeed, or Facebook.

Therefore, content providers try to increase their direct revenues in order to build a stable revenue stream mainly by means of digital paywalls. For example, German market data on the digital news publishing market from 2014 to 2018 confirm that direct sales revenues show a much stronger growth ( $€ 112$ million to $€ 359$ million) than advertising revenues ( $€ 238$ million to $€ 325$ million) (PricewaterhouseCoopers 2019). Promising additional revenue, digital paywalls could also stall the cannibalization of offline editions by free digital editions. Additionally, these instruments could increase the value of the website's individual users for advertisers. However, besides the ongoing competition with free content alternatives on the Internet, consumers show a general lack of willingness to pay for online content compared to the physical equivalent, which makes the pricing of access to online content an uncertain endeavor (Berger et al. 2015). Furthermore, pricing online content can decrease viewership and drive down online advertising revenue (e.g., Chiou and Tucker 2013). Therefore, content providers must configure their paywall solutions carefully to improve their content monetization. Hence, content providers have to understand implementation and configuration possibilities to establish a viable revenue model in the digital economy.

In this catchword article, we introduce digital paywalls as an instrument to address the content monetization issue. We differentiate between paywall systems and paywall solutions to account for both the technological and economic aspects of digital paywalls. A paywall system allows for mediating the access to content by distinguishing between users who have paid for the access and those who have not. We describe the overarching design of paywall systems with related modules in a stylized content providers' IT architecture (Sect. 2). Building on the paywall system, content providers can configure various paywall solutions to monetize their online content. Accordingly, a paywall solution is the combination of specific economic configuration parameters, namely access restriction options and pricing options (see Sect. 3). In addition, the use of Machine Learning (ML) provides new opportunities and challenges when configuring paywall solutions, which we debate (see Sect. 4). In conclusion, we summarize existing knowledge on digital paywalls, and propose directions for future research. Overall, IS research on digital paywalls is still sparse, with several economic and technological aspects being unexplored. The IS discipline can contribute by addressing the challenges regarding the design of the paywall's system architecture, as well as the configuration possibilities of paywall solutions and their economic impact on content providers' direct (sales) and indirect (advertising) revenue streams across online and offline channels.

\section{Technological Functionality of Paywall Systems}

The system architecture of media companies commonly consists of a content management system (CMS), a customer relationship management (CRM) system, an enterprise resource planning (ERP) system, and a website or application software. ${ }^{1}$ Within the CMS, an editorial system provides functions to plan, create, and change content, while a content repository system provides functions to store and retrieve content, and a publication system transforms content into different output formats. The CRM system documents historical customer data and provides functions to manage customer relationships. Lastly, the ERP system with integrated media sales enables the administration and consolidation of customer and transactional data.

Based on existing systems and their application programming interfaces, content providers can integrate paywall systems in their system architecture that are either dependent or independent and either integrated or adjacent to the CMS (see Fig. 1). The grey-shaded modules represent the core systems, which are independent of a content provider's attempt to restrict access to online content. The black modules are the essential components of a paywall system, namely (1) access control management, (2) subscription and access policy management, and (3) user interface.

The main functionalities of the access control management $(A C M)$ module are to identify individual users on the website (i.e., authentication) and to implement the decision whether they are permitted or not to access requested content (i.e., authorization). Initially, a user requests access to a specific content unit on the website. The ACM module uses technologies like cookies or JavaScript to track and identify the individual user by collecting a number of available identification parameters ( $\mathrm{Pa}-$ padopoulos et al. 2019). If the user is eligible to access the content, a server delivers the content page and access is granted. Otherwise, the ACM module directs the user to a login page to verify the user's credentials. In the case of a successful verification, the authorized user can access the requested content. However, if the verification is not

\footnotetext{
$\overline{1}$ We focus on core systems and do not consider the adjacent systems and modules, such as the specifics of advertising technologies, data warehouses, or data interfaces.
} 
Fig. 1 Stylized content providers' system architecture with an integrated paywall system

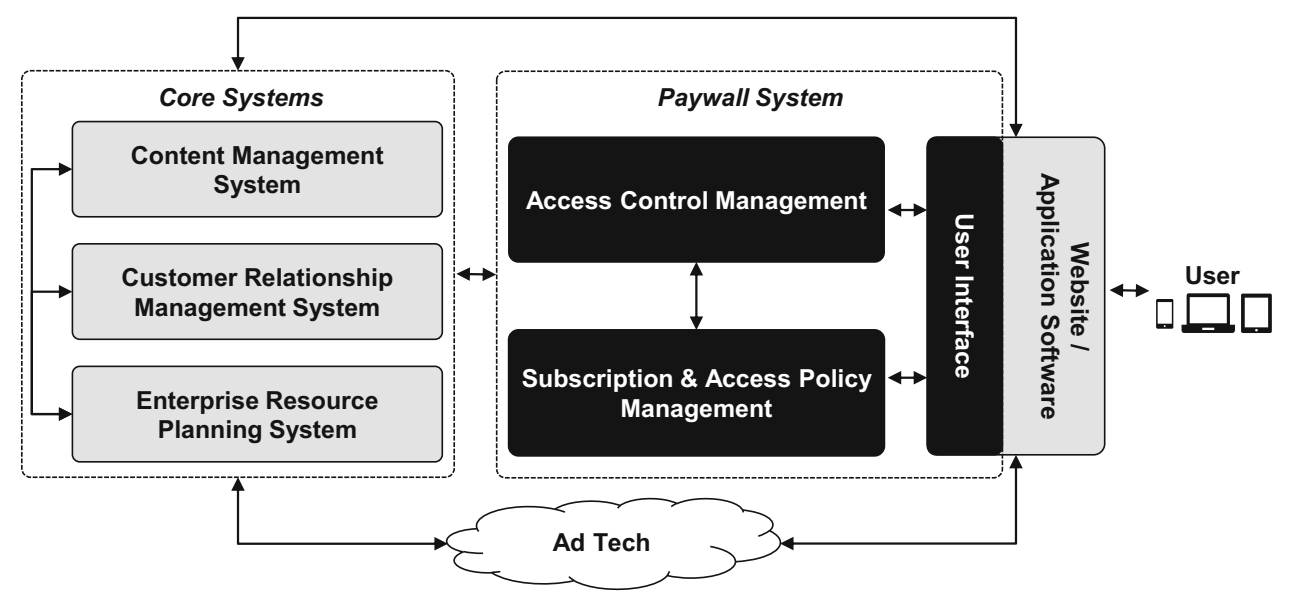

successful, the ACM module blocks the access to the requested content until the user is authorized.

The subscription and access policy management (SAPM) module manages the ACM module's operations by setting up the access restriction and pricing rules (see Sect. 3). An interface to the CMS is necessary, if the access to specific content units is supposed to be restricted. The access restriction and pricing rules can be orchestrated by humans or automated by algorithms based on various data sources (see Sect. 4). Accordingly, an interface to the content providers' accessible data sources is required to define the rules and subsequently provide relevant information to the ACM. The payment interface is a commonly integrated submodule of the SAPM, which provides different payment methods or links to payment service providers. The payment interface exchanges subscriber and transactional data with the CRM system and the ERP system.

The user interface facilitates a frictionless user experience of the internal processes of the paywall system (e.g., a paywall page with login and registration options; a selfservice-area to update personal data and contracts).

The implementation of a paywall system provides content providers with new opportunities to leverage data, for example anonymous data from non-paying users and personalized data from registered users. Content providers could track the entire customer journey on their website, from the first visit to the actual selection of subscription options and re-purchase behavior. However, these opportunities produce new challenges. To use the data intelligently, content providers need to store, link, and integrate the data streams in a central database, and manage additional information accordingly. In addition, content providers need to operate website analytics tools to adapt the configuration of a paywall solution according to user activity. Based on the paywall system, content providers can configure paywall solutions to monetize their content. In the next section, we present the fundamental configuration parameters of paywall solutions.

\section{Configuration of Paywall Solutions}

Once content providers have established the technological capabilities to restrict access to their content, they can subsequently configure the access restriction and pricing rules in the SAPM module of the paywall system. Online content is an information good which consumers need to experience in order to evaluate their expected benefits and costs (Lopes and Galletta 2006; Shapiro and Varian 1998). Accordingly, content providers follow different strategies to offer either free content, paid content, or a combination of the two. Additionally, the cost structure of digital information goods with a high fixed cost but almost zero variable cost for distribution raises challenges regarding what is priced (i.e., paying per use of the content, per period, or offering bundles with the offline edition) and how to set prices (i.e., based on costs or other metrics such as consumers' perceived value). Building on the literature on revenue models (e.g., Veit et al. 2014) and pricing models (e.g., Skiera et al. 2005), we present a framework to classify paywall solutions by their most relevant economic configuration parameters, namely access restriction options and pricing options (see Fig. 2).

Whereas access restriction options specify the mechanism to access content (see Sect. 3.1), pricing options define how the access to this content is priced (see Sect. 3.2). By selecting at least one option on each of the two layers, paywall solutions can be classified according to their respective functions.

\subsection{Access Restriction Options}

Overall, we distinguish between three access restriction options, which follow different rules to decide whether a user can access the requested content or not.

First, content providers can place a donation request on their website whenever a user accesses the content. This mechanism does not restrict the access to content but asks 


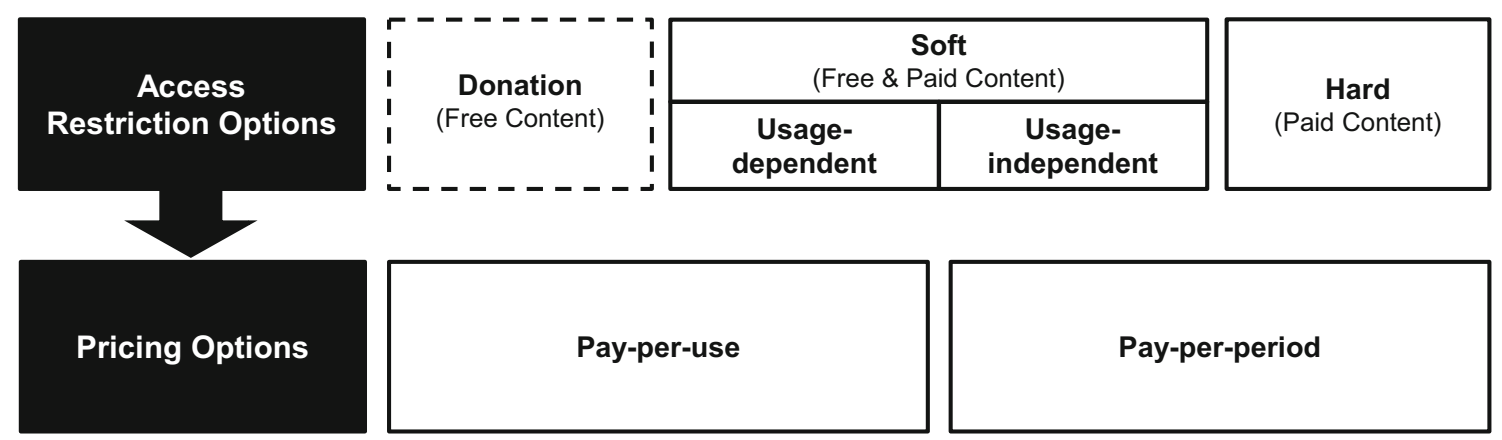

Fig. 2 Core configuration parameters of a paywall solution

users for a voluntary payment. This can be either a request at the end of the free content consumption or a permanent display ad on the website. Content providers who follow a donation strategy may believe that their content on the website needs to be open and accessible to anyone (Reuters Institute 2018). A widely used economic pricing mechanism for voluntary payments is called 'Pay What You Want'. This mechanism asks consumers to pay any price they like, including zero (Schmidt et al. 2014). Examples of media companies who successfully follow this strategy are The Guardian and Wikipedia.

Second, content providers can implement a soft access restriction, which allows at least some free content on the website. This access restriction rule is based on a freemium model, which simultaneously offers a limited free version (i.e., free content) and a premium version with additional benefits such as functionality or higher quality (i.e., paid content) (Anderson 2009). By providing two versions of the content, the media companies are able to differentiate prices between consumers with heterogeneous valuations for content (Lambrecht and Misra 2017). Theory distinguishes between price differentiation with and without selfselection. In price differentiation without self-selection, the seller divides buyers into different groups, offering varying prices for each group (e.g., discounts for students). In contrast, in price differentiation with self-selection, the seller offers the same product in different versions at different prices, and allows consumers to decide which offer suits them. The second mechanism is closely related to the soft access restriction of a paywall solution, in which content providers offer free content and let consumers decide if they are willing to pay for the content behind the paywall. The soft access restriction can further distinguish whether or not the access restriction depends on the individual usage behavior of consumers on the website, that is a usage-dependent or usage-independent access restriction.

In a usage-dependent access restriction, content providers give consumers the freedom to choose their preferred free content within a pre-defined allowance. Therefore, news publishers have no control over what content individual consumers actually consume (Halbheer et al. 2014). Accordingly, the access restriction rule is dependent on the individual usage (i.e., browsing behavior) of consumers on the website. The access restriction rule can be based on the quantity of requested contents' access (e.g., a certain number of news articles or movies per month), or the time a consumer spends on browsing the website's content (e.g., a certain duration of free content consumption per day), before a payment for the unlimited access is required (Chiou and Tucker 2013; Wilkinson 2013). An example of a usage-dependent access restriction is The New York Times's 'metered' paywall, which allows free access to a certain number of news articles per month.

In contrast, the usage-independent access restriction limits the access to content by offering a pre-defined set of free content, which is independent of the individual usage of consumers. However, the consumption behavior of consumers on the website can indirectly affect the selection of free content, because the differentiation between free and paid content is often determined by the content providers' purported value of content for the audiences (Olsen and Solvoll 2018). With this access restriction option, content providers are able to manipulate the free content strategically, that is they can decide what content to offer for sampling (Halbheer et al. 2014). Widely used classification rules to differentiate between free and paid content are based on the breadth of offered free content (e.g., access restriction to specific genres), the objective quality of free content (e.g., production costs; resolution of content; exclusiveness of content itself), or consumers' preferences (e.g., cumulative demand for specific content; temporal access restrictions) (Aral and Dhillon 2017; Chiou and Tucker 2013; Li et al. 2019; Oh et al. 2016). An example of a usage-independent access restriction is the French news publisher Le Monde, which offers specific content (e.g., exclusive news articles) as paid content.

Third, content providers can place a hard access restriction on their website to restrict the access to the entire content by solely offering paid content. In this option, consumers cannot access any content without 
certain content-related restrictions. However, content providers usually allow consumers to access teasers of their content free of charge (e.g., first paragraphs of a news article). The Times, for example, launched a hard paywall on their news website in 2010 , believing that their quality content is worth paying for.

A paywall solution requires a certain rule to restrict access to the content providers' online content (see Fig. 2). The execution of access restriction rules can be based on a variety of content and user data. Particularly, the soft access restriction rules can be combined in the configuration of paywall solutions. Optimizing the access restriction rules based on data-driven decisions (e.g., consumers' usage patterns) using ML is becoming increasingly important (see Sect. 4). Besides the core access restriction rules, content providers can differentiate their free and paid content by additional features, such as refraining from advertising in the paid content, device specific access restrictions, or free access via referrers such as social media platforms. Moreover, many content providers offer a free trial access to the entire content and allow consumers to bypass the access restriction options for a certain period.

\subsection{Pricing Options}

The pricing options of a paywall solution define how the access to the media companies' content is priced. More specifically, these rules determine the quantity of content units (e.g., the number of articles, songs, movies) that users can access with a payment, as well as the duration of the access period. We analyze the overarching pricing decision that content providers face when intending to monetize their online content. For this purpose, we focus on the pricing rules related to the configuration of a paywall solution.

Essentially, we differentiate between pay-per-use, that is a usage-dependent tariff, and pay-per-period, namely a usage-independent tariff or flat-rate. In theory, rational consumers are expected to choose the tariff that will maximize consumer surplus conditional on their beliefs about individual usage, namely the quantity of content they are going to consume. However, several studies show that consumers prefer a pay-per-period to a pay-per-use mechanism even though their resulting surplus is lower (Lambrecht and Skiera 2006). The pay-per-use mechanism requires users to pay a small fee, a micropayment, for each unit of paid content they want to consume. An advantage of this pricing mechanism for consumers is that they exclusively pay for content that interest them. For content providers, these micropayments have the advantage that the users' entry barrier is much lower compared to higher, usage-independent pricing mechanisms. In contrast, the pay-per-period mechanism allows consumers to access an unlimited quantity of content units in a pre-defined period. Hence, it provides consumers with a flat-rate for a specific period. The advantages of this pricing mechanism compared to micropayments are the higher comfort of a single payment for the user and the improved revenue predictability for content providers. The pay-per-period mechanism can be differentiated into a single payment for a pre-defined period, and a subscription, which is an agreement for a recurring payment over a pre-defined contract duration. Content providers often reward loyal subscribers who opt for longer contract durations with discounts on the recurring payments. Many content providers use a mixed pricing option, that is a pay-per-use and a pay-per-period option to maximize direct revenue from online content by exploiting consumers' heterogeneous valuations.

\section{Use of Machine Learning in Paywall Solution Configurations}

Digital paywall systems' functionalities can be enhanced by ML to adjust and combine the solution's configuration parameters based on various data points. Hereby, digital paywalls allow content providers (1) to individualize access restriction and pricing options based on users' behavior on the website and (2) to identify improved classification schemes with respect to whether specific content should be free or paid content. Specifically, ML allows varying access restriction and pricing rules by recognizing patterns in user behavior data over time. The ML model first needs to be trained with historical data. Accordingly, the model learns about differences in data patterns between objects (e.g., users, content units) which reveal certain reactions (e.g., user conversion), and objects which do not reveal the examined reactions. Based on these patterns, the model can determine the optimal paywall configuration for a specific user. The users' interaction with the paywall then serves as a basis for the ongoing improvement of the paywall configuration. Figure 3 depicts a stylized workflow from data extraction and storage to model training and the adaptive digital paywall. Apart from data analytics competencies, a fundamental requirement for using ML in optimizing digital paywall configurations is a solid data infrastructure to extract and synchronize various data streams such as anonymous visitor data, personalized data from registered users and customers, content data, and additional contextual data.

In the following, we describe three applications of ML to transform static paywalls configured by humans into dynamic paywalls configured by algorithms. First, content providers can model users' conversion probability score based on behavioral data (e.g., visit recency and 


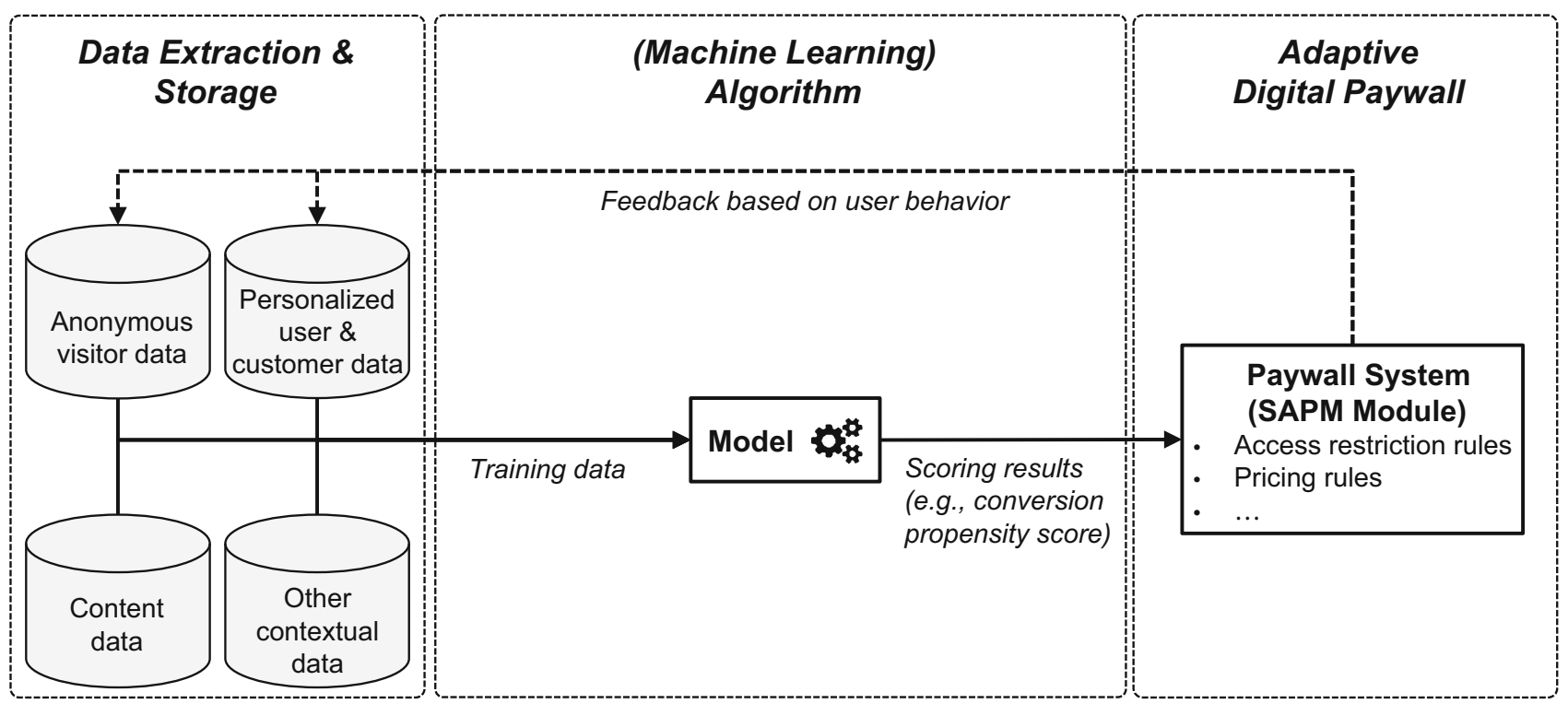

Fig. 3 Stylized workflow of using machine learning in configuring paywall solutions

frequency). The model learns about (individual) user's content consumption and conversion patterns and dynamically adapts the paywall solution's configuration (e.g., quantity of free content units). Second, content providers can use ML models to identify which content is most suitable as free or paid content. A content value score can be predicted by content-related metrics, such as the content's theme, and usage related-metrics, such as stickiness (i.e., the duration a user reads the specific content). The model could identify content that drives conversions and classify it accordingly. Third, paywall systems can simultaneously optimize content classification and the individualization of the paywall solution's configuration. Additionally, content providers could feed ML algorithms with contextual data, such as the time of the day or external events (e.g., elections). The result can be a dynamically adjusting digital paywall solution, which presents different (free and paid) content to individual users or segments.

Besides the individualization of the paywall solution's configuration and classification of the content providers' offer, ML can also be used to optimize communication strategies (e.g., personalized newsletters), to engage customers by improving the audience's website experience (e.g., personalized front-page), and to support content creation (e.g., reporting emerging topics). However, increasing the use of ML and automation by means of artificial intelligence can also have negative consequences. First, by managing ML models as 'black boxes', content providers delegate control to algorithms. Content providers increase the risk of filter bubbles if they allow algorithms to decide which content is displayed to a specific audience. Second, content providers face challenges with regulations such as the General Data Protection Regulation (GDPR) or the ePrivacy Regulation, which prohibit cookie-based tracking of users without their explicit consent. From the consumer's perspective, identifying the usage of personal data (e.g., by product recommendations) could lead to intensified perceptions of privacy concerns, subsequently increasing the probability of website abandonment by consumers.

\section{Prior Research and Avenues for Future Work}

Digital paywall research has its origins in business model research on the monetization of online content (e.g., Clemons 2009) and optimal content strategies (e.g., Fan et al. 2007).

One research stream analytically studies when media companies can benefit from offering free content, paid content, or both. Fan et al. (2007) conclude that media companies should offer paid content if the content quality is high and access costs are low. In addition, Halbheer et al. (2014) show that advertising effectiveness can determine the optimal content monetization strategy. Another research stream looks empirically at the consequences of a paywall introduction. Most studies leverage quasi-experimental settings of paywall introductions and find a negative impact on website traffic (Aral and Dhillon 2017; Chiou and Tucker 2013; Cook and Attari 2012; Pauwels and Weiss 2008) and word of mouth activities (Oh et al. 2016). In contrast, charging for access to online content not only provides direct revenue but also protects own paid channels from self-cannibalization (Pattabhiramaiah et al. 2019) and 
enhances control over the substitutive effects of crosschannel demand (Aral and Dhillon 2017).

Although initial paywall research has been conducted, very little is known about the impact of paywall solution's configuration on user behavior. According to Aral and Dhillon (2017), a decrease in the quantity and an increase in the breadth of free content can increase the probability of a non-paying user becoming a subscriber. Furthermore, Lambrecht and Misra (2017) show that it can be optimal for media companies to offer more free content during periods of high demand. To the best of our knowledge, only Davoudi et al. (2018) investigate the effect of adaptive paywall solutions by modeling the restriction rule execution based on users' costs and the utilities of articles. They show that the adaptive mechanism can outperform static mechanisms by applying the proposed method on a real data set of a Canadian news publisher.

Future research should advance our understanding of digital business models for content providers and of the role of IT in developing and managing content monetization strategies. Subsequently, we outline three promising directions for future research.

- The impact of paywall solution's configuration on consumer behavior in pre-conversion (acquisition) and post-conversion stages (engagement and retention) Researchers could empirically examine this relationship by conducting experiments or analyzing secondary data obtained from content providers. Additionally, research could focus on important and relevant moderators and mediators of this relationship, for instance by examining content, consumers, markets, and additional paywall characteristics to generate insights on optimal paywall configurations.

- The effect of online content monetization strategies across media products and their online and offline channels While studies in the context of news content exist, IS research could examine the applicability of digital paywalls beyond this type of content. For example, the German video streaming platform Joyn recently implemented a usage-independent access restriction for specific serials and movies on their website. Additionally, IS research could focus on content providers' multi-channel activities, as well as on bundling the offerings of one company or between companies, which aim to increase the value of the content providers' paid content. Moreover, platform characteristics of media companies' websites need to be further examined to optimize content monetization strategies within this multi-sided market.

- The impact of technological innovations and their applicability on paywall systems and resulting paywall solutions' configuration Current trends in ML or blockchain technology influence digital paywalls by providing new system functionalities and related architecture designs. Recent general developments in the media industry include the personalization and recommendation of content, as well as the automation of content creation (Newman 2019). Accordingly, questions arise regarding consumers' attitudes toward automated processes, privacy concerns based on content providers' data usage, and threats to freedom of opinion and the content providers' role of informing the public (e.g., filter bubbles) as consequences of the use of ML.

Insights into these topics can assist content providers to better prepare themselves for their future in the digital economy. Owing to its interdisciplinary foundations, the IS discipline is particularly well positioned to advance this field of research. The paid content market is growing and in this process continuously bringing forward innovative approaches to solve the content providers' monetization dilemma. In this relatively young research area of IS, the BISE community could set new ground rules for strategies to sell media products based on digital paywall approaches.

Acknowledgement Open Access funding provided by Projekt DEAL.

Open Access This article is licensed under a Creative Commons Attribution 4.0 International License, which permits use, sharing, adaptation, distribution and reproduction in any medium or format, as long as you give appropriate credit to the original author(s) and the source, provide a link to the Creative Commons licence, and indicate if changes were made. The images or other third party material in this article are included in the article's Creative Commons licence, unless indicated otherwise in a credit line to the material. If material is not included in the article's Creative Commons licence and your intended use is not permitted by statutory regulation or exceeds the permitted use, you will need to obtain permission directly from the copyright holder. To view a copy of this licence, visit http://creativecommons. org/licenses/by/4.0/.

\section{References}

Anderson C (2009) Free: the future of a radical price. Random House, London

Aral S, Dhillon P (2017) Digital paywall design: implications for subscription rates \& cross-channel demand. SSRN working paper 2906530. https://ssrn.com/abstract=2906530. Accessed 7 Nov 2018

Berger B, Matt C, Steininger DM, Hess T (2015) It is not just about competition with "free": differences between content formats in consumer preferences and willingness to pay. J Manag Inf Syst 32:105-128. https://doi.org/10.1080/07421222.2015.1095038

Chiou L, Tucker C (2013) Paywalls and the demand for news. Inf Econ Pol 25:61-69. https://doi.org/10.1016/j.infoecopol.2013. 03.001

Chyi HI (2012) Paying for what? How much? And why (not)? Predictors of paying intent for multiplatform newspapers. Int $\mathrm{J}$ Media Manag 14:227-250. https://doi.org/10.1080/14241277. 2012.657284 
Clemons EK (2009) Business models for monetizing internet applications and web sites: experience, theory, and predictions. J Manag Inf Syst 26:15-41. https://doi.org/10.2753/MIS07421222260202

Cook JE, Attari SZ (2012) Paying for what was free: lessons from the New York Times Paywall. CyberPsychol, Behav Soc Netw 15:682-687. https://doi.org/10.1089/cyber.2012.0251

Davoudi H, An A, Zihayat M, Edall G (2018) Adaptive paywall mechanism for digital news media. KDD '18: the 24th ACM SIGKDD international conference on knowledge discovery \& data mining. ACM, New York, pp 205-214

Fan M, Kumar S, Whinston AB (2007) Selling or advertising: strategies for providing digital media online. J Manag Inf Syst 24:143-166. https://doi.org/10.2753/MIS0742-1222240305

Halbheer D, Stahl F, Koenigsberg O, Lehmann DR (2014) Choosing a digital content strategy: how much should be free? Inf J Res Mark 31:192-206. https://doi.org/10.1016/j.ijresmar.2013.10. 004

Lambrecht A, Misra K (2017) Fee or free: when should firms charge for online content? Manag Sci 63:1150-1165. https://doi.org/10. $1287 / \mathrm{mnsc} .2015 .2383$

Lambrecht A, Skiera B (2006) Paying too much and being happy about it: existence, causes, and consequences of tariff-choice biases. J Mark Res 43:212-223. https://doi.org/10.1509/jmkr.43. 2.212

Li H, Jain S, Kannan P (2019) Optimal design of content samples for digital products and services. J Mark Res 56:419-438. https:// doi.org/10.1177/0022243718823169

Lopes AB, Galletta DF (2006) Consumer perceptions and willingness to pay for intrinsically motivated online content. J Manag Inf Syst 23:203-231. https://doi.org/10.2753/MIS0742-1222230209

Newman N (2019) Journalism, media, and technology trends and predictions 2019. https://reutersinstitute.politics.ox.ac.uk/ourresearch/journalism-media-and-technology-trends-and-predic tions-2019. Accessed 28 Mar 2019

Oh H, Animesh A, Pinsonneault A (2016) Free versus for-a-fee: the impact of a paywall on the pattern and effectiveness of word-ofmouth via social media. MIS Q 40:31-56
Olsen RK, Solvoll MK (2018) Bouncing off the paywall understanding misalignments between local newspaper value propositions and audience responses. Int $\mathrm{J}$ Media Manag 20:174-192. https://doi.org/10.1080/14241277.2018.1529672

Papadopoulos P, Snyder P, Livshits B (2019) Another brick in the paywall: the popularity and privacy implications of paywalls. https://arxiv.org/abs/1903.01406. Accessed 4 Sept 2019

Pattabhiramaiah A, Sriram S, Manchanda P (2019) Paywalls: monetizing online content. J Market 83:19-36. https://doi.org/ $10.1177 / 0022242918815163$

Pauwels K, Weiss A (2008) Moving from free to fee: how online firms market to change their business model successfully. J Market 72:14-31. https://doi.org/10.1509/JMKG.72.3.014

PricewaterhouseCoopers (2019) German entertainment and media outlook 2019-2023. https://www.pwc.de/de/technologie-med ien-und-telekommunikation/german-entertainment-and-mediaoutlook-2019-2023.html. Accessed 5 Dec 2019

Reuters Institute (2018) Digital news report 2018. http://media. digitalnewsreport.org/wp-content/uploads/2018/06/digital-newsreport-2018.pdf?x89475. Accessed 26 March 2019

Schmidt KM, Spann M, Zeithammer R (2014) Pay what you want as a marketing strategy in monopolistic and competitive markets. Manag Sci 61:1217-1236. https://doi.org/10.1287/mnsc.2014. 1946

Shapiro C, Varian HR (1998) Information rules: a strategic guide to the network economy. Harvard Business Press, Boston

Skiera B, Spann M, Walz U (2005) Erlösquellen und Preismodelle für den Business-to-Consumer-Bereich im Internet. Wirtschaftsinformatik 47:285-293. https://doi.org/10.1007/BF03254916

Veit D, Clemons E, Benlian A, Buxmann P, Hess T, Kundisch D, Leimeister JM, Loos P, Spann M (2014) Business models: an information systems research agenda. Bus Inf Syst Eng 6:45-53. https://doi.org/10.1007/s12599-013-0308-y

Wilkinson EJ (2013) News media outlook 2014: navigating the minefield. https://www.inma.org/report-detail.cfm?pubid=178. Accessed 1 Apr 2019 